\title{
Thermal transition from a disordered, 2D network, to a regular, 1D, Fe(II)-DCNQI coordination network
}

Jonathan Rodríguez-Fernández, ${ }^{1}$ Yang Wang, ${ }^{2,3}$ Manuel Alcamí, ${ }^{2,3}$ Fernando Martín, $, 2,3,4$ Roberto Otero, ${ }^{1,3}$ José M. Gallego, ${ }^{5}$ and Rodolfo Miranda ${ }^{1,3}$

1 Departamento de Física de la Materia Condensada, Universidad Autónoma de Madrid, Cantoblanco, 28049-Madrid, Spain

2 Departamento de Química, Módulo 13, Universidad Autónoma de Madrid, 28049Madrid, Spain

3 Instituto Madrileño de Estudios Avanzados en Nanociencia (IMDEA-Nanociencia), Cantoblanco, 28049-Madrid, Spain

4 Condensed Matter Physics Center (IFIMAC), Universidad Autónoma de Madrid, 28049Madrid, Spain

5 Instituto de Ciencia de Materiales de Madrid (CSIC), Cantoblanco, 28049-Madrid, Spain 


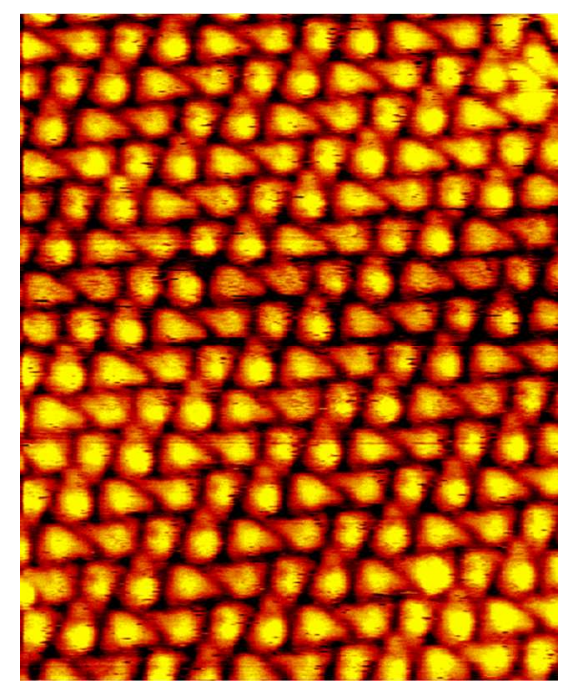

Figure 1. STM image (92 $\AA$ x $115 \AA$ ) taken after depositing DCNQI on Ag(111) at room temperature. The syn conformation is clearly visible in this image. 